\title{
1 A Physical Effort-Based Model for Pedestrian Movement in 2 Topographic Urban Environment
}

3 Eliyahu Greenberg

4 Faculty of Architecture and Town Planning, Technion IIT, Haifa, Israel.

5 greeliyahu@campus.technion.ac.il

6 Asya Natapov

7 Centre for Advanced Spatial Analysis, University College London, London, UK.

$8 \quad$ a.natapov@ucl.ac.uk

9 Dafna Fisher-Gewirtzman

10 Faculty of Architecture and Town Planning, Technion IIT, Haifa, Israel.

11 ardafna@ar.technion.ac.il 
1 A Physical Effort-Based Model for Pedestrian Movement in 2 Topographic Urban Environment

3 Abstract This paper presents a topography-sensitive cognitive model for analysis and prediction of pedestrian movement in urban settings. Topography affects visibility and therefore the spatial awareness of pedestrians. It also accentuates the role of physical effort

6 during travel and route selection. The existing models fall short in their reference to these 7 issues.

8 A thorough description of the proposed model is followed by a validation - the model was

9 tested against two existing models in three case studies in Haifa and Jerusalem, Israel. The

10 proposed model outperformed the others in the steeper parts of the case studies. Future model

11 development is discussed.

12 Keywords: topography, physical effort, axial map, pedestrian movement, spatial analysis.

\section{1. Introduction}

14 This paper presents a novel cognitive model for the analysis of pedestrian movement 15 in an urban setting. The model incorporates both mutual visibility and physical effort 16 as cognitive bases. Both apply to any pedestrian movement and are greatly affected by 17 steep topography, where the role of physical effort is especially accentuated. This type 18 of environment and its influence on cognitive perception impede the effectiveness of cognitive models that do not consider both factors. The objective of this work is the development of a topography-sensitive cognitive model that can help planners make more informed decisions.

The need for such a model arises from a gap between the terms in which the built environment is described by users and the tools professionals use to create it. While human experience is extensively discussed, the way places make people feel and the atmosphere they possess, there are only a few robust qualitative methods that rely on human cognition to translate this discussion into concrete spatial terms. It can be argued that experience and intuition can offer a limited solution to this problem, especially in smaller projects, but this solution is often inadequate. This is especially true since the design of complex projects is a difficult task that only gets harder the more the project's size and complexity increase (Karimi, 2012). 
4 Cognitive mapping techniques and simulations were created to address the 5

\subsection{Cognitive mapping techniques}

\subsubsection{Motivation and overview}

abovementioned issues. They are intended to present information about spatial arrangements and their usage that cannot be easily derived from conventional spatial representations, such as plans and sections. This is achieved by representing space using methods derived from human cognitive perception, or at least, some aspect of this complex phenomenon.

Producing mapping methods based on cognition requires understanding some principals of human spatial perception and navigation. It has been found that the more junctions (decision points) there are along a path, the longer it is perceived to be (Sadalla and Staplin, 1980). The number of turns along a path has the same effect on the perception of its length (Sadalla and Magel, 1980). Similar effects are observed when referring to maps rather than to the environment itself (Thorndyke, 1981). The angularity of paths affects spatial orientation, right angles are generally less confusing than oblique ones and there is a bias towards judging angles to be closer to a right angle than they really are (Montello, 1991; Sadalla and Montello 1989; Moar and Bower, 1983). This can cause descriptions of both locations and paths to defy the Euclidian properties of space (Moar and Bower, 1983). The importance of orientation (the direction one faces) for performing tasks where one is required to locate elements in space is found to be inconsistent for relatively short distances but significant for large-scale geographic knowledge (Montello, 1991; Sholl, 1987). When people learn new environments, they quickly remember the locations of a few landmarks and paths between them, and over time their knowledge of the place is expanded with more paths and junctions, but not additional landmarks (Evans, Marrero and Butler, 1981). These findings are supported by Montello's claim that the acquisition of environmental knowledge is done in a process of quantitative accumulation and refinement of metric and non-metric, global and local knowledge simultaneously (Montello, 1998). This, combined with the difficulty of mapping landmarks, might explain why, despite much

\section{Background}


work on landmarks in the field of cognitive mapping, they are generally absent from the analytical cognitive-based models this paper examines.

Mutual visibility has thus far been the most common cognitive basis for mapping and analysis techniques. Some techniques designed to describe space like the Isovist (Benedikt, 1979). Others are concerned with the perception of space. For example, the work regarding perceived density which produces progressively complex models (Fisher-Gewirtzman, 2017; Fisher Gewirtzman and Wagner, 2003). Some techniques, like the Visibility Graph Analysis (VGA) analyse the functioning of spaces (Turner et al, 2001). There are other works that are concerned with movement in larger systems, analysing streets and junctions to predict movement (Jiang 2006, 2005; Jiang and Claramunt 2004, 2002). Moreover, there are yet others that attempt to model movement in a different manner from those top-down approaches by introducing socalled 'agents' that represent discreet entities (like a person or a car) into an environment and observing their behaviour. Each agent acts according to a limited set of rules and the collective behaviour of the agents emerges in a bottom-up process, modelling anticipated movement (Koutsolampros and Varoudis, 2017; Penn and Turner, 2004; Turner and Penn, 2002; Batty, 2001).

\subsubsection{Cognitive mapping of movement in urban systems}

This work is concerned with the analysis of street networks and the associated pedestrian movement. The planning of street networks is a long_-term affair as many systems around the world remain virtually unchanged for centuries. As such, the design should deal with the most general characteristics of a network, like its structure and spatial relations. These characteristics imbue street networks with the quality, flexibility and longevity of a robust urban fabric; a fabric that supports the creation of opportunity that cities are so cherished for, while leaving more detailed design to be determined according to changing needs. A group of techniques, collectively known as Space Syntax, presents a compatible candidate for a mapping and analysis technique of street networks - the Axial Map (Hiller and Hanson, 1984). In general, an Axial Map is created from a plan of the environment by dividing the space of the entire system into a minimal number of discreet spaces with convex shapes in a plan and then connecting all those spaces with a minimal number of axes, representing lines of sight. The intersecting axes should cover the entire system. These maps can be displayed in 
1 a graph where every axis is represented by a node and every intersection is represented 2 by an edge (Figure 1).

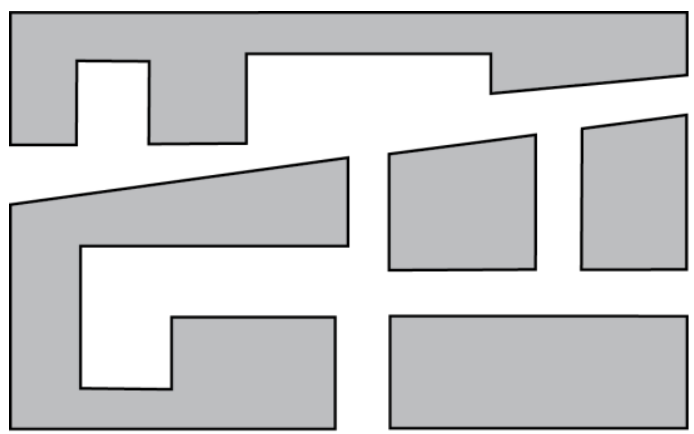

1. Area plan

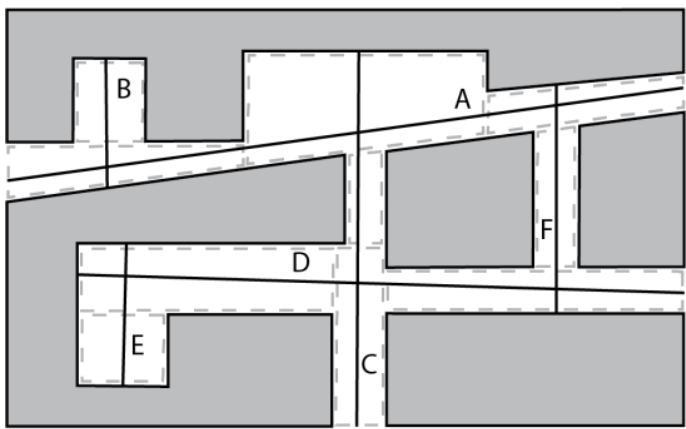

3. Axial map

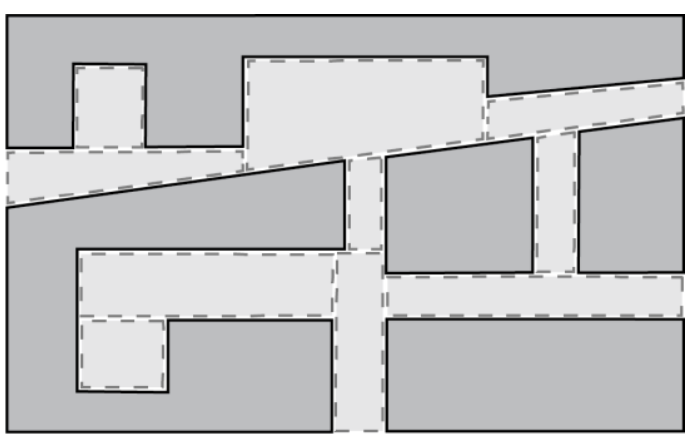

2. Convex map

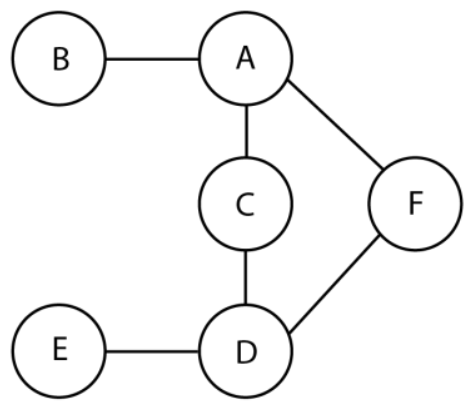

4. Representation as a graph

Figure 1. The stages of axial map construction.

The angular segment model is an attempt to improve on the traditional topological axial map (Conroy Dalton, 2003; Dalton, 2001; Turner, 2001). This is a model that takes into account incident angles between axes to determine the weights assigned to different edges in a graph when calculating centrality measures: the sharper the turn

9 the higher the weight, with right angles having a weight of 1 . The graph representation of this model is weighed, meaning that the cost of traveling along an edge varies between edges, which affects the lengths of shortest paths and therefore also centrality measure calculations. This model is considered by many to provide more accurate

13 predictions of pedestrian movement than the traditional axial map (Conroy Dalton,

14 Hölscher and Turner, 2012).

\section{2.1.3. From graphs to quantitative measures}

16 The graph that represents an axial map can be analysed for various centrality measures

17 that correspond to a wide variety of social phenomena (Hillier, 1996a; Hillier et al, 18 1993). One such important centrality measure is Integration, or Closeness, as it is 
called in some publications. It is defined as the inverse of the mean shortest distance from the space in question to all other spaces in the system (Hillier and Hanson, 1984). Distance is calculated according to the relevant model: topological distance for axial maps, angular distance for segment maps, etc. It represents how easy it is to navigate to a space from all other spaces (Hillier, 1996b). Equation 1 describes the way integration is calculated.

$$
\text { Integration of an axis }=\frac{\text { number of axes }-1}{\text { sum of shortest paths from the axis to all other axes }}
$$

The abovementioned calculation describes global integration; global in the sense that this measure is calculated relative to the entire system in question. It is possible to calculate centrality measures, including integration, on a more local scale. The calculation itself is identical, the only difference is that the sum of the shortest paths incorporates paths only up to a certain distance. This distance is referred to as a radius. Some specific radii have been demonstrated to be more useful than others when describing certain things. There have also been suggestions for more complex methods, such as decay functions, for local indices calculation to describe complex phenomena (Conroy Dalton and Dalton, 2007).

\subsection{The Challenge of Topography}

The model proposed in this paper is meant to overcome the challenges presented to the pedestrian by steep topography. Topography, or a sloped walking surface, has a substantial effect on human perception and behaviour in an urban context. It can be summed in two main ways:

1) Topography can potentially block lines of sight and limit the spatial awareness of pedestrians.

2) The added effort of walking on steep slopes can serve as a consideration for pedestrians when deciding which path to choose (or whether to walk at all).

An example of limited visibility was demonstrated when Jiang and Claramunt's model of mutually visible junctions as nodes in a graph (2002) was applied to a neighbourhood in Haifa built on hilly topography. The visibility of junctions as 
described by three methods, junctions as nodes, line of sight analysis (FisherGewirtzman, 2012) and voxel-based analysis (Fisher-Gewirtzman, Shashkov and Doytsher, 2013), was compared. The study showed that when a junction was located at the edge of a hill, Jiang and Claramunt's method overestimated the visibility of junctions because it ignored the blocking effect of topography on visibility, while the other two methods were more consistent because they were based on 3D visual information on the environment (Fisher-Gewirtzman and Natapov, 2014).

The effect of physical effort is discussed in chapters 2.2.1 and 2.2.2.

A cognitive mapping model that takes these issues into account is required to better represent urban environments built on steep topography.

\subsubsection{The effects of physical effort}

The effects of physical effort, especially the increased effort of walking on inclined surfaces, plays a major role in human cognitive interpretation of the environment. Physical effort can be an organizing element in the perception of space (Proffitt et al, 2003) but it is also closely related to visual information (Zadra and Proffitt, 2016). The perception of geometrical traits, like distance, is affected by the effort needed to traverse it (Witt, Proffitt and Epstein, 2010). The perception of distance is also scaled in energetic terms, rather than visual ones (Zadra, Weltman and Proffitt, 2016), meaning that people perceive things as near or far according to their own ability to reach them. This, combined with the fact that climbing a slope is harder than walking on a flat plane might explain why humans consistently estimate distances on slopes to be greater than they really are (Stefanucci et al, 2005). Although the decision to take a sloped path is made in advance based on visual cues, the incentives for and against travelling the path also depend on physical effort. Humans can visually judge the accessibility of sloped surfaces fairly well and can predict their own ability of traversing a slope (Kinsella-Shaw, Shaw and Turvey, 1992). Although city streets are unlikely to be steep enough to prevent human travel, they might still discourage it or shorten the distance people are willing to walk (Sun et al, 2015).

\subsubsection{Existing topography-sensitive models}

For all of its successes, the axial map and its derivatives are still 2D representations derived from 2D information that depict the structure of a 3D environment perceived 
through 3D vision. However, it has representative clarity, flexibility and a robust logic making it suitable for modification. For these reasons, the axial map was chosen as the basis for a new model that enables a more accurate mapping of environments built on slopes.

Asami et al. (2003) successfully addressed the issue of visibility limited by topography in axial maps by dividing the axial lines at peak points in the topography. Yet, this model only deals with visibility limitations and does not consider physical effort as a factor. The weight system used in this model differs from the one used in angular segment analysis but is still based on mutual visibility, rather than physical effort. Asami et al. tested their model against the axial map in the old city of Istanbul. They found their model to offer some improvement in predicting the location of commercial centres over the traditional method, despite taking into account only the visual effects of topography.

An attempt to integrate physical effort as a factor in shortest-path choice in axial maps was done by Nourian et al. (2015). The model they proposed translated many factors, including physical effort, into time measured in seconds. The shortest path was that which required the shortest time to traverse. Although such a method considers many factors and offers a coherent measuring scale, namely time, it has limitations: physical effort is modelled only by the effect slope has on movement speed. This can be treated as an inaccuracy from a cognitive standpoint as it was shown that distance is scaled in energetic terms (Zadra, Weltman and Proffitt, 2016). Only uphill paths are modelled as having special considerations of movement, while downhill slopes were treated as flat. Another weakness is the use of time as the measure of shortest path, because the angularity of paths has been found to correlate with distance estimation but not travel time estimation (Sadalla and Magel, 1980).

This research is aimed at developing a model based on the axial map, dealing with both problems described above: limited visibility and additional physical effort in movement. First, a topography-sensitive model that tackles both problems was developed. In order to evaluate the validity and accuracy of the proposed model, it was compared to other methods of integration analysis in axial maps, namely the traditional topological approach described by Hillier and Hanson (1984), as well as a 
variant of analysis based on the angle of incident (Asami et al, 2003). The comparison is done using 3D models representing existing urban environments built on steep topography in Haifa and Jerusalem, Israel.

\section{The Physical Effort Model (PEM)}

The proposed model has a two-stage operation: the first stage overcomes the limitations on visibility caused by topography and the second one deals with representing the relative physical effort of traveling up and down slopes. First, the model redraws the input axial map in a way that represents actual lines of sight, making sure topography does not block them. Then, the axes in the processed map are assigned weights based on their slopes that represent the relative difficulty of travel along them. These weights, in turn, affect centrality measure calculations derived from this map to better represent actual pedestrian movement.

For technical reasons that concern the way the model conducts calculations, all models discussed in this paper are axial segment models rather than axial line models (Dalton, 2001; Turner, 2001). The logic behind this decision, what it implies and how the model deals with those implications are described in section 3.2. An axial segment map is created with the axial lines broken at intersections with other axial lines, creating axial segments. Redundant segments can then be omitted. This means that any space that requires more than a single axial line to map will require more axial segments than axial lines for this purpose. For example, a simple T-shaped space can be mapped using two axial lines, but will require three axial segments; the larger and more complex the space the greater the difference in number.

The PEM needs to receive an axial segment map of an assessment area as input, together with an axial segment map of a 'buffer' area around it. The buffer area and its' purpose are explained in section 4. The PEM can work without the buffer area segments, but this will adversely affect the accuracy of the analysis. Thus the initial axial segment map is input into the model in four groups: segments from the test area, segments from the test area that represent flights of stairs, segments from the buffer area and segments from the buffer area that represent stairs.

The segments that represent stairs should be drawn in a different colour from other segments because the model identifies types of segments by that trait. Segments 
2 of the model.

3 The model can also conduct analyses as the topological and angular models would.

4 One should select the desired type of analysis when running the model: Topological, 5 angular or physical. Only the physical analysis type is explained in length in this

6 section. The two other types of analysis were developed by others and therefore

7 described in the introduction.

8 The PEM is a CAD based model, executed in Grasshopper for Rhino with Python 9 scripting.

\subsection{Problem 1: Limited visibility}

Axial segments, just like axial lines, represent lines of sight that can be blocked by topography. Therefore, the $2 \mathrm{D}$ axial segment map must be redrawn according to the topography to represents actual lines of sight. In order to do so, the PEM requires as input a 3D model of the topography and a corresponding 2D axial segment map. Whatever the format the topographic information is in, be it a digital topographic model (DTM), topography lines in CAD or any other format, it must be converted into a surface (or mesh) before being input into the PEM.

The PEM divides the axial lines into short, equal, segments. The length of the segments is controllable and should be set according to the resolution of the available topographic data. For example, if the topographic information is available as a DTM with a 5 meters by 5 meters raster resolution the length of the segments should preferably be somewhat smaller than 5 meters. The division points are then projected onto the topographic model. As shown in Figure 2, only the peak points, the points at the edges of a plateau and the end points of original segments are retained to draw the new 3D segments (Greenberg et al, 2017). 


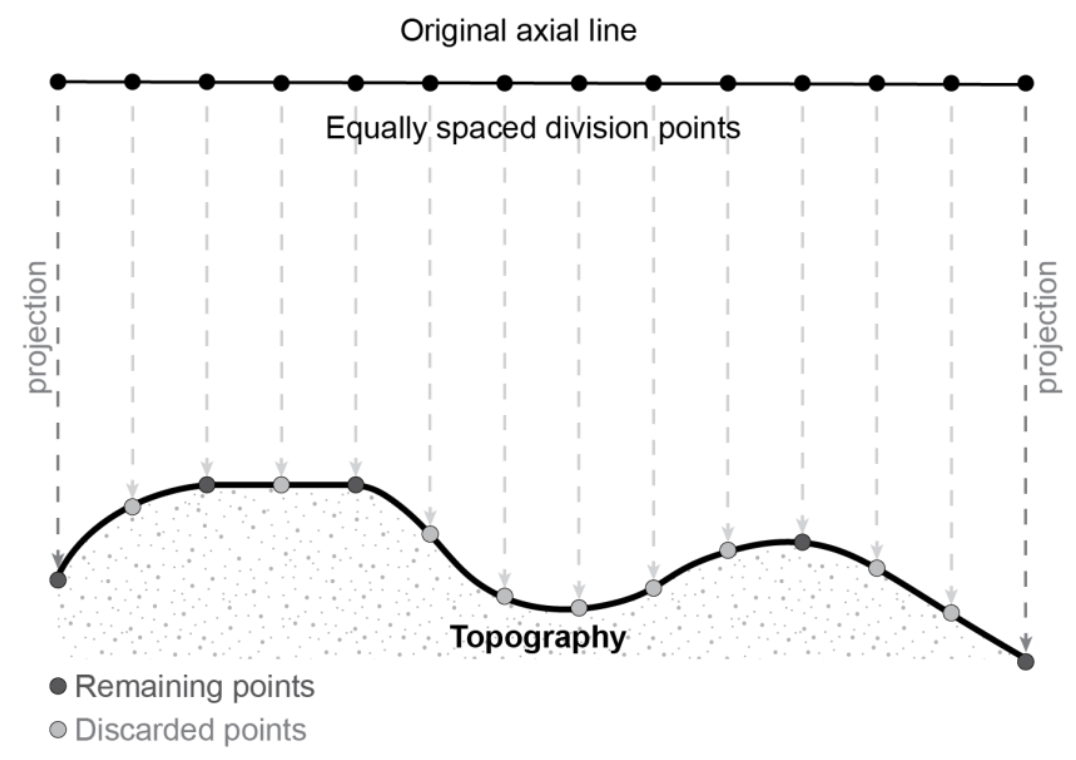

Figure 2. A section view. Only the dark points are retained for future processing in the model, others are discarded.

4 This process allows one to draw the 3D segments in a topography-sensitive way, but 5 might still be excessive. In such a process, any variation in height is enough to break 6 an axial segment in two. This, of course, provides exaggerated sensitivity to terrain 7 that can both damage the accuracy of the analysis as well as slow down calculations. 8 To avoid these problems, a way to determine which features actually affect spatial 9 awareness is needed. For this, typical human eye height is used. This metric varies in 10 different populations, so the exact height can be set according to the geographic 11 location of the case study and statistical information regarding the average height of 12 the population there.

13 The points received from the previous stage are tested to eliminate 'bumps' along the 14 3D segments that are deemed insignificant. The PEM checks if the point after the point 15 on the 'bump' along the 3D segment can be seen from human eye height above the 16 point on the other side of the 'bump', and vice versa. If both points, the one before the 17 'bump' and the one after it, can be seen in this manner the point on the 'bump' is 18 eliminated (Figure 3). Only the remaining points are then connected to create new 3D 19 segments. The resulting 3D segments are then input into the next stage of the analysis. 

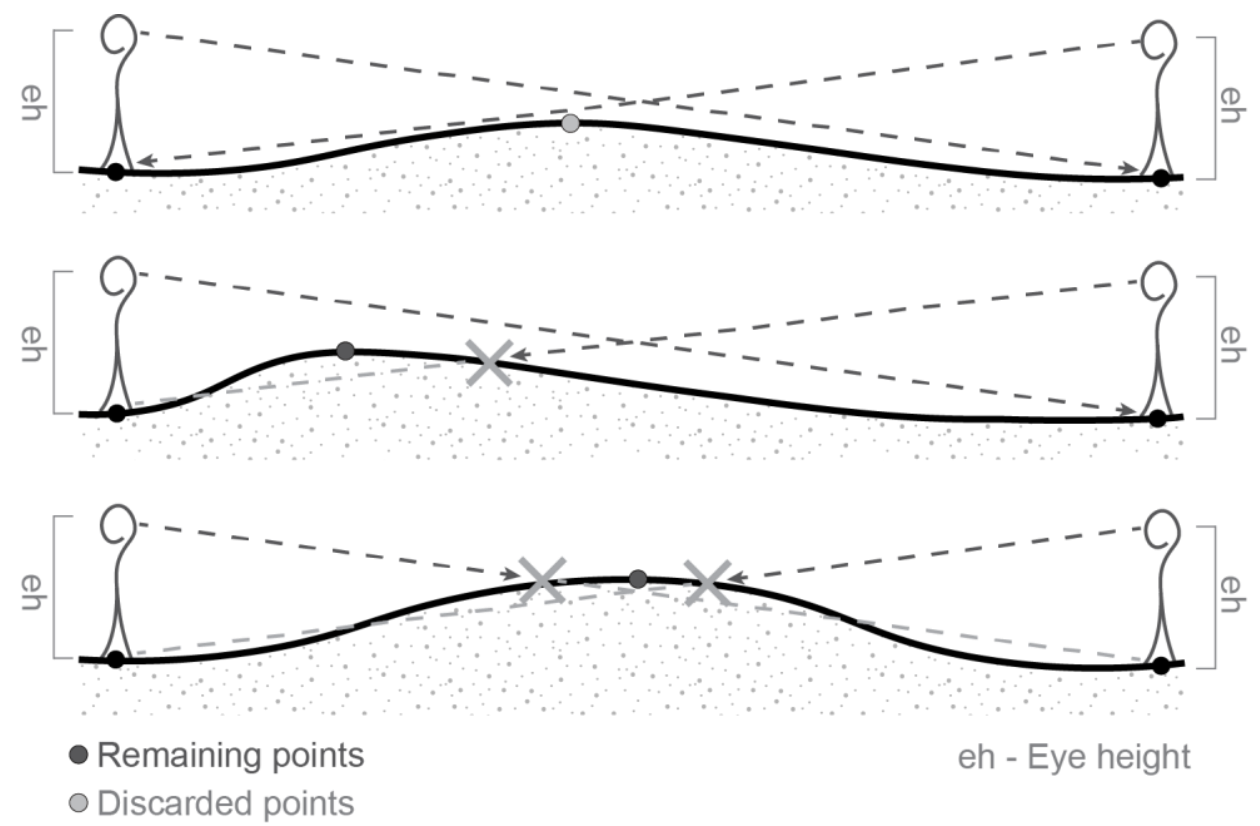

Figure 3. A section view.

3 Segments representing stairs are treated differently from other types of segments. They

4 are not divided into smaller parts prior to projection onto the topographic model and

5 therefore are not tested to eliminate redundant 'bumps', either (Figure 4). The

6 projection is merely used to determine the slope of the stairs, which is important for

7 the next stage. The reason for this is that stairs are used to connect different levels over

8 shorter distance than is otherwise possible and are, therefore, unlikely to go first up

9 and then down in a manner that will block a line of sight.

Original axial line

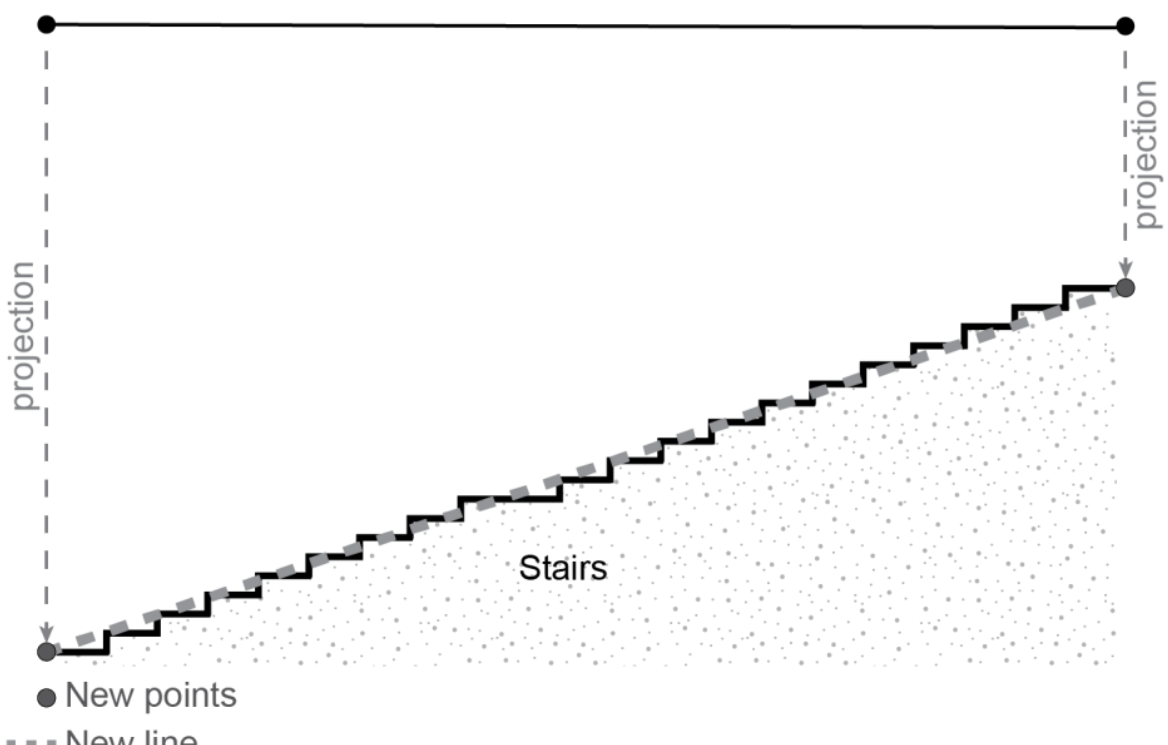

Figure 4. A section view. With stairs, only the average slope of the entire staircase is considered. 
Winding staircases that are meant to bridge large differences in elevation over a short distance are a special case in which a staircase is regarded as a single segment only if the ends of the entire staircase (not just one flight) are mutually visible.

\subsection{Problem 2: Physical effort}

Integrating the effect of physical effort into the model requires a breakaway from the exclusivity of mutual visibility as the cognitive basis for axial maps (Bafna, 2003). The PEM suggests differentiating between walking downhill or uphill and walking in plane in physical - rather than visual - terms.

\subsubsection{Weights of physical effort}

Integrating physical effort into the PEM is done by attaching weights, or costs, to every 3D segment. Since it is easier to walk downhill than uphill, each 3D segment gets two different weights attached to it - one for each direction. However, the manner in which these weights are determined is open to debate. Unfortunately, most studies that deal with the parameters that affect pedestrian path selection ignore slope as a contributing factor (Golledge, 1995; Seneviratne and Morral, 1985). The effects of slope were modelled indirectly in studies that sought to optimize path selection to the shortest travel time parameter by using an inverse of the Hiking Function described by Tobler (1993; Equation 3) multiplied by some constant (Whitley and Hicks, 2003). In another study, an attempt was made to improve the accuracy of this method by normalizing this function according to paths in nature, as they are thought to represent an evolution of human movement free of the constraints of the built environment which can be arbitrary and force certain paths (Pingel, 2010). However, it is argued in this article that methods that take only the effects of slope on walking speed to determine costs are insufficient since they show almost similar costs for traveling a steep slope uphill and downhill. Downhill travel indeed requires careful movement and therefore slows down speed, but such a model ignores the fact that downhill travel is done when the traveller works together with planetary gravity toward a common goal, instead of against it. This difference is deemed significant and the costs of travel should represent that. Therefore, the model developed in this work takes physical effort itself into account. This variable is represented by metabolic power which describes the amount of metabolic energy required to walk on a given slope for a given length of time (the 
4

energy itself required to traverse the entire sloped path depends on the length of the path as well). The weights used for the PEM in this paper are derived from the value of metabolic power calculated using a quadratic equation for power as a function of walking velocity (see Equation 2; Al-Widyan et al., 2017).

$$
P=A v^{2}+B v+C
$$

$$
C=1.5 \mathrm{~W}+2(\mathrm{~W}+\mathrm{X})(\mathrm{X} / \mathrm{W})^{2}
$$

$\mathrm{P}$ - power, $\mathrm{v}$ - walking speed in $\mathrm{m} / \mathrm{sec}, \mathrm{W}$ - individual weight in $\mathrm{kg}, \mathrm{X}$ - load carried in $\mathrm{kg}$, $\alpha$ - the slope in degrees, $\mu$ - terrain factor, defined as 1 for free walking.

Walking velocity is derived from the slope of the axis using an equation described by Tobler (1993; Equation 3).

$$
v=6 e^{(-3.5|\tan \alpha+0.05|)}
$$

$\mathrm{v}$ - walking speed in $\mathrm{km} / \mathrm{h}, \alpha$ - the slope in degrees. The walking speed units must be turned into $\mathrm{m} / \mathrm{sec}$ (by dividing $\mathrm{v}$ by 3.6) before being input into equations 2 or 4 .

The values derived from equation 2 are used as an extra cognitive weight in addition to a turn and should therefore be in a range between 0 and 1 . For this reason, the absolute values in the equation are not important, only its behaviour. This enables a stripping of component $\mathrm{C}$ of the equation as it is not affected by slope and does not alter the behaviour of the function. Both components A and B contain some expression multiplied by $\mu(W+X)$ which means it does not affect the relationship between those two components and can be discarded as well. This results in the much simplified equation 4.

$$
P=1.5 v^{2}+0.35 v \tan \alpha
$$


Equation 2 was derived from cases where walking speed was constant and only the other variables changed. This makes it only partly suitable for our case because speed is not predetermined but may be dependent on slope. The simplified equation 4 will be adapted for this purpose. As it stands, inputting equation 3 into equation 4 results in an absurd case: the steeper the slope - the lower the metabolic power. This contradicts common sense as climbing steep slopes is not physically easier than walking on plane or downhill. Equation 4 has the exact opposite behaviour from reality. To overcome this contradiction the equation must be inversed, just like equation 3 was inversed when used as a basis for cost calculation (Pingel, 2010; Whitley and Hicks, 2003). This is possible because equation 3 is used as input for equation 2 to create equation 4 . Dividing 0.1 by the result of equation 4 results in a value range of roughly 0 to 1 in a range of realistic inclination between 0 and 30 degrees (50\% slope). The weight for uphill walking will be $1+0.1 / P$ and the weight for downhill walking $1-0.1 / P$, to represent the difference in effort according to the direction of travel. This also inverts the equation in a way that is consistent with reality, at least in the sense that climbing steeper slopes requires higher energy expenditures.

The weighting method described above is an experimental one. Other methods that take physical effort as a direct source of the weights assigned to segments can be considered, be it Pingel's (2010) method or any other that might be conceived.

Two main types of paths are considered: regular paths and stairs. Staircases are often built in the urban context to bridge height differences over a shorter distance compared to a ramp or sidewalk. The downside in the use of stairs is that they are generally less accessible than flat inclined surfaces as they are very hard (or impossible) to use for people whose mobility is limited for whatever reason, whether temporarily or permanently: physical fitness, old age, injury, disease, driving a cart or a stroller, carrying cumbersome weight - any of these cases can make using a staircase very difficult. Thus, 3D segments that represent staircases receive additional penalties to their weights, making them higher, to account for the reduced accessibility of such paths to certain groups. This penalty can be set by the operator according to the specific parameters of the analysis but should be compatible with the range of possible integration scores. 
2 The graph for the 3D segment map in this model is weighted and directional. The

3 weights are used when calculating the shortest path in the graph utilizing a realization

4 of a greedy shortest path algorithm (Dijkstra, 1959). The algorithm varies from the

5 original in being implemented on a directional graph; therefore, it must be decided

6 which weights to use in each specific path. Making this decision requires the direction

7 of travel to be known. The weight to be used is determined by comparing the height

8 of the point of intersection of axial segments, which is an end point of both segments,

9 to the second end point of the next axial segment along the path. If the intersection

10 point is higher than the other point, the weight for downhill travel is used and vice

11 versa (Figure 5). In the PEM, a 3D segment in a valley will have different integration,

12 for example, than the same segment in an identical system on a ridge (Figure 6).

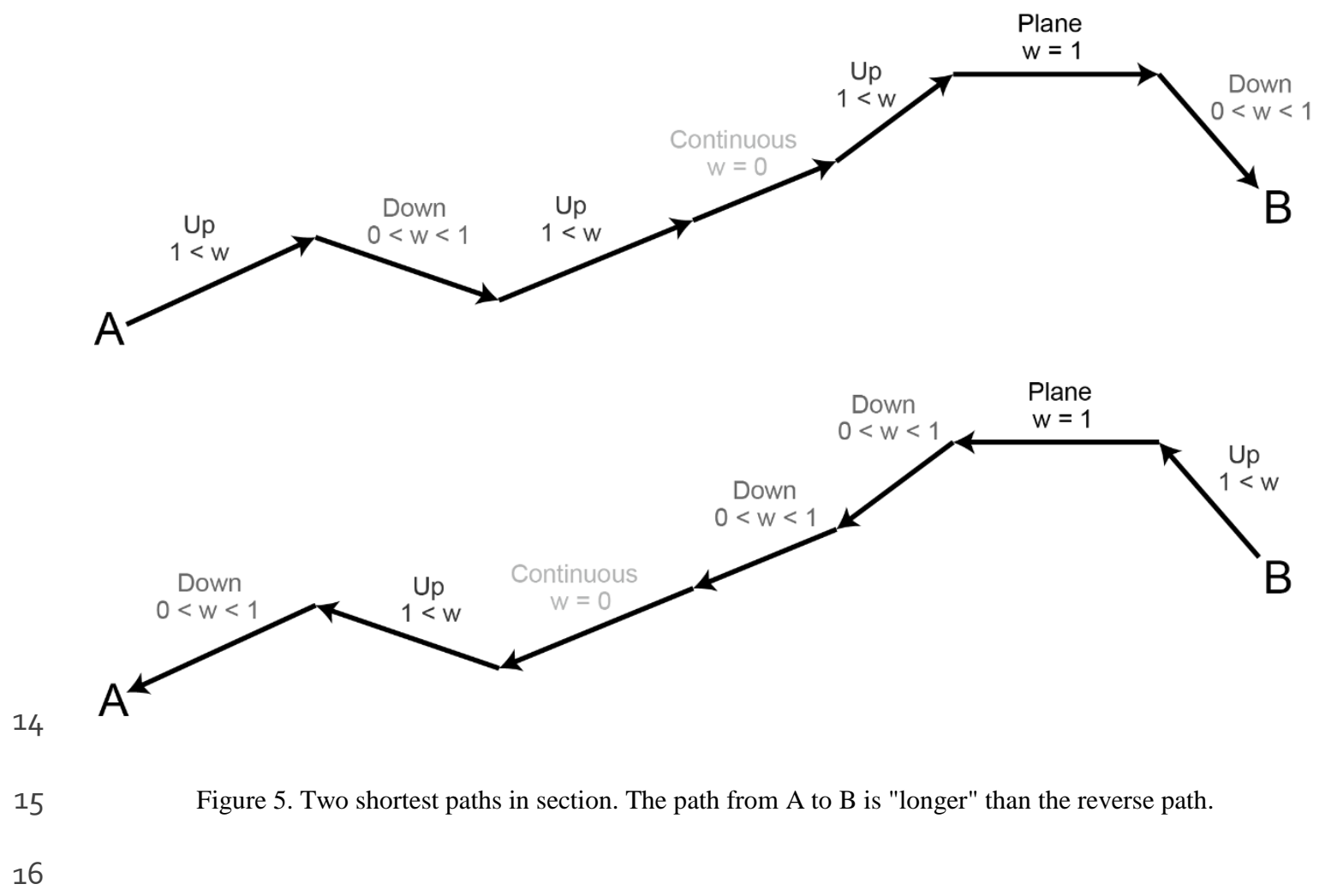




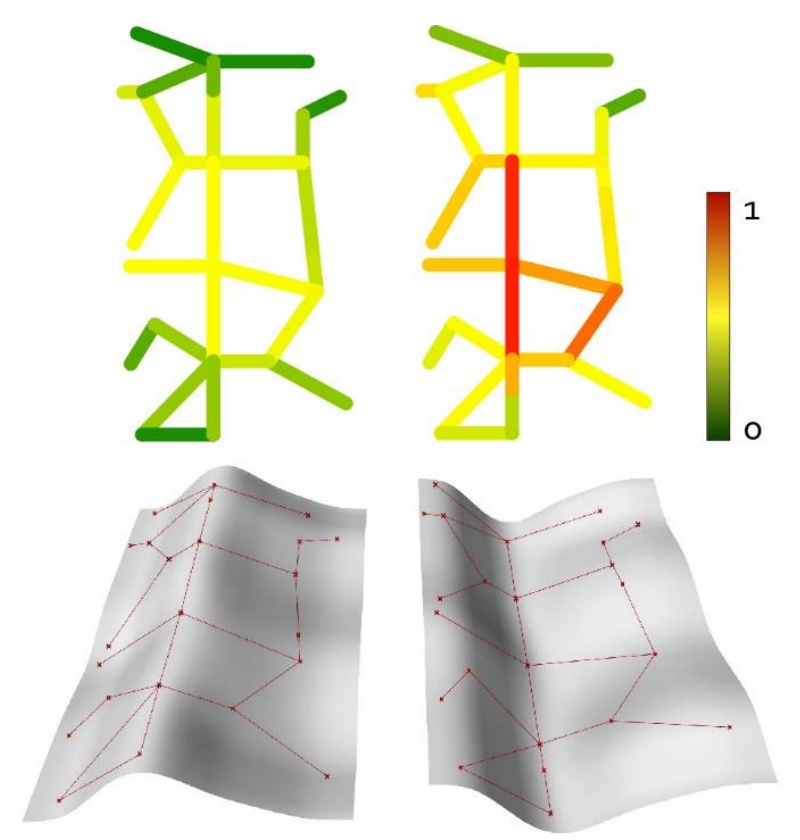

Figure 6. Identical systems - different integration. The road that goes along the watershed is less integrated than the road that runs through the valley.

4 Axial segments rather than axial lines are used as an input for the PEM because there

5 is a very likely possibility that upon projection of the axial line unto the topographic

6 model two axial lines that intersect in 2D will no longer intersect in 3D creating an

7 artificial disconnection (Figure 7). This further division generates two problems. The

8 first problem is that going along what is essentially a single axial line might now be

9 treated as a turn. This is solved by checking whether two segments along a path are

10 continuous and assigning no weight to the continuing segment in this specific path

11 calculation. The second problem occurs when calculating indices in the graph, as the

12 number of axial lines (now segments) in the system is artificially inflated. This

13 problem is solved by considering all continuous axial segments as a single line for the

14 purpose of calculating indices. 


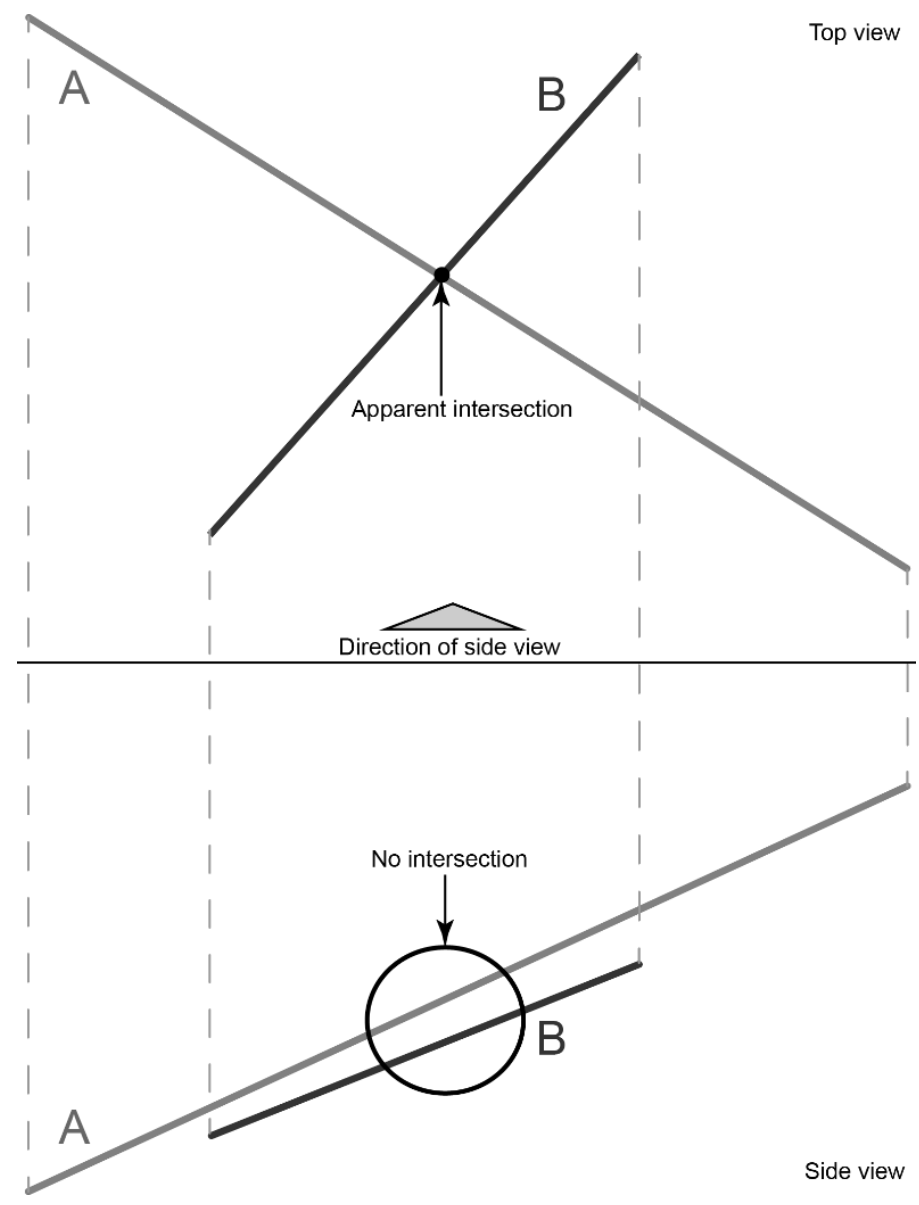

Figure 7 . When transitioning from $2 \mathrm{D}$ to $3 \mathrm{D}$ by projection unto a topographic model two lines that used to intersect might now be disjoint, but it cannot be seen in top view. Such cases would result in grave misrepresentations.

The analytic rigor sought in this method can be, under certain circumstances, a weakness. The issue results from a conflict between the perfect accuracy of an analytical model and the limited accuracy of human cognition and perception (Dutta, 1988). It was shown that the absolute accuracy of analytical mapping can sometimes highlight differences in the environment that are small and insignificant or misrepresent the human interpretation of it, which can result in faulty representation of phenomena (Ratti, 2004). One such problem relates to the question whether two axial lines are continuous. A case of perfect geometrical continuity is obvious enough, but what about very subtle incidents? It can be argued that from a pedestrian's perspective a turn of only a few degrees or a small change in slope makes no difference at all and is not even registered as such (Frith, 2017; Sun et al, 2015; Montello and Frank, 1996). For this reason, the model has an angular tolerance feature that enables one to set a maximum angle (in degrees) between two 3D segments below which the 8 two lines would be considered continuous. 


\subsection{PEM Summary}

To sum up, the PEM needs the following input data to run an analysis: an axial segment map, a corresponding topographic model, an analysis mode (topological, angular or physical), a radius for analysis (set at $O$ for a global calculation), a segment length for initial division of axial segments, an angular tolerance value, a list of colors corresponding to the list of axial segments, the color of the axial segments representing staircases, the value of the weight penalty for stairs. A general flowchart of the model is shown in Figure 8.

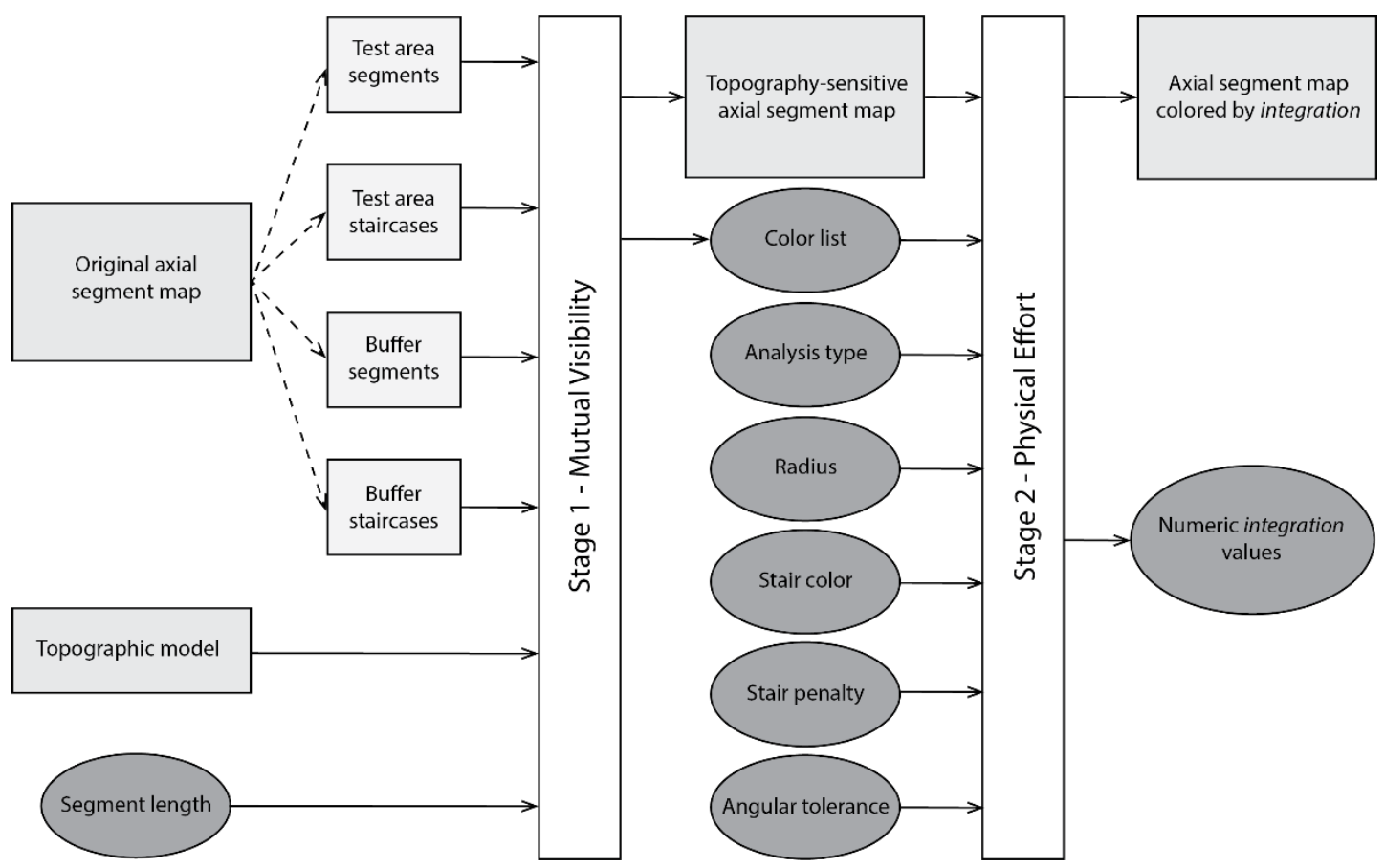

Figure 8. A general flowchart of the model's operation, inputs and outputs. Rectangular shapes indicate geometric data, like CAD drawings. Elliptical shapes indicate numbers, strings or data structures containing them.

\section{PEM Assessment}

In order to assess the performance of the PEM it was compared to the other two axial segment models described above: The topological model and the angular model. All models go through the first stage of the PEM (redrawing segments according to topography) so the comparison is only between the analysis modes. The reason it is important for a cognitive model based on mutual visibility to have its' lines actually represent lines of sight is self-evident and hard to argue against. The second stage of the model is much more open to scrutiny and therefore is the one to be evaluated. 
For the assessment, three real urban environments with significant topography were selected as case-studies. Each case study was mapped and analyzed for integration using all three models. The integration values were compared to the density of businesses along the corresponding segments to see if and how well they correlate. Business-density is the number of businesses along a segment divided by the length of that segment. This measure, rather than a simple business count, is used to negate the advantage longer segments have in their ability to accommodate more businesses compared to shorter ones. The use of businesses-density as an approximation of pedestrian movement is based on the mutual reinforcement between spatial configuration, pedestrian movement and commerce (Hillier 1999; Hillier et al. 1993).

This method has some disadvantages. First, it is less accurate than the direct approach - pedestrian counts. Another problem is that a small business and a large business register as equal in the count despite the different volumes of pedestrian flows that sustain them.

The case studies for the validation of the PEM should be roughly neighbourhoodseized, because it is best suited for analysis at this scale. As the PEM bases itself on human perception of the immediate environment and spatial memory it can be applied at any scale, but on larger scales radii should be applied to get meaningful results. Although street network analysis can be done on a metropolitan scale (or even larger), it is not as relevant for pedestrian movement analysis - movement on foot on these scales is a very different phenomenon and is not as restricted spatially as pedestrian movement in urban environments. There is a body of work that deals with spatial memory and orientation, perception of distance and physical effort in natural settings (Egorova, 2018; Pingel, 2010; Okabe, Aoki and Hamamoto, 1987).

\subsection{Parameters and Important Considerations}

The evaluation included global, radius 3 and radius 4 integration analysis, with an angular tolerance value of $1^{\circ}$ and a weight penalty of 0.1 for staircases (only applicable in the 'physical effort' mode). Radius 3 was included as it was found to often correlate well with pedestrian movement volumes in axial maps. Radius 4 was included because weights above 1 are common in both the PEM and the angular model of Asami et al (2003). 
1 It was previously demonstrated that incomplete data about the routes available to

2 pedestrians can distort the analysis of pedestrian networks (Chin et al, 2007). To avoid

3 this, pedestrian paths were completed using OpenStreetMap as well as site

4 observations to create a comprehensive documentation of a pedestrian network.

5 Another problem for any analysis of a real-world network is the 'edge effect'. As street

6 networks in reality are not strictly discreet systems, the borders of the area of analysis

7 are defined somewhat arbitrarily. Yet the world outside the area of analysis still affects

8 the world inside it and the movement patterns within. In such cases, centrality

9 measures in the analysis area are somewhat distorted because they disregard the effects

10 of elements outside it. This effect gets more pronounced the closer a certain node (a

11 3D segment, in this case) is to the edge of the area of analysis (Okabe and Sugihara,

12 2012). In order to mitigate distortions to centrality measure calculations stemming

13 from proximity to the edges of area of analysis, additional axes outside the test areas

14 are included in the analysis, but their measures are not analysed (as they are not reliable

15 enough) and they serve as an inert 'buffer' for the actual test area (Gil, 2017; Penn et

16 al, 1998; Hillier et al 1993). This is not a definitive solution, but it diminishes the 'edge

17 effect' significantly. The buffer size in the analysis is set at 5, which means that five

18 topological steps are added to the axial segment map beyond the boundary of the

19 assessment area (Figure 10, where the 'buffer' segments can be seen around the 20 assessment areas of the case studies).

21

22

\subsection{The Case Studies}

The assessment environments for the comparison that was selected are the Hadar neighborhood in the city of Haifa and the City Center and Nahlaot neighborhoods in Jerusalem (Figure 9).

Hadar is a Garden-City neighbourhood built on a steep continuous slope and many staircases connecting different levels in the neighbourhood.

Jerusalem City Center serves as the central business district (CBD) of Jerusalem and is built mostly in traditional European city blocks without many courtyards or much vegetation. 
1 Nahlaot is a conglomeration of small sub-neighborhoods, each comprised of just a few 2 streets or blocks. Most buildings are low-rise stone structures that are either 3 concentrated around courtyards or along narrow alleys.

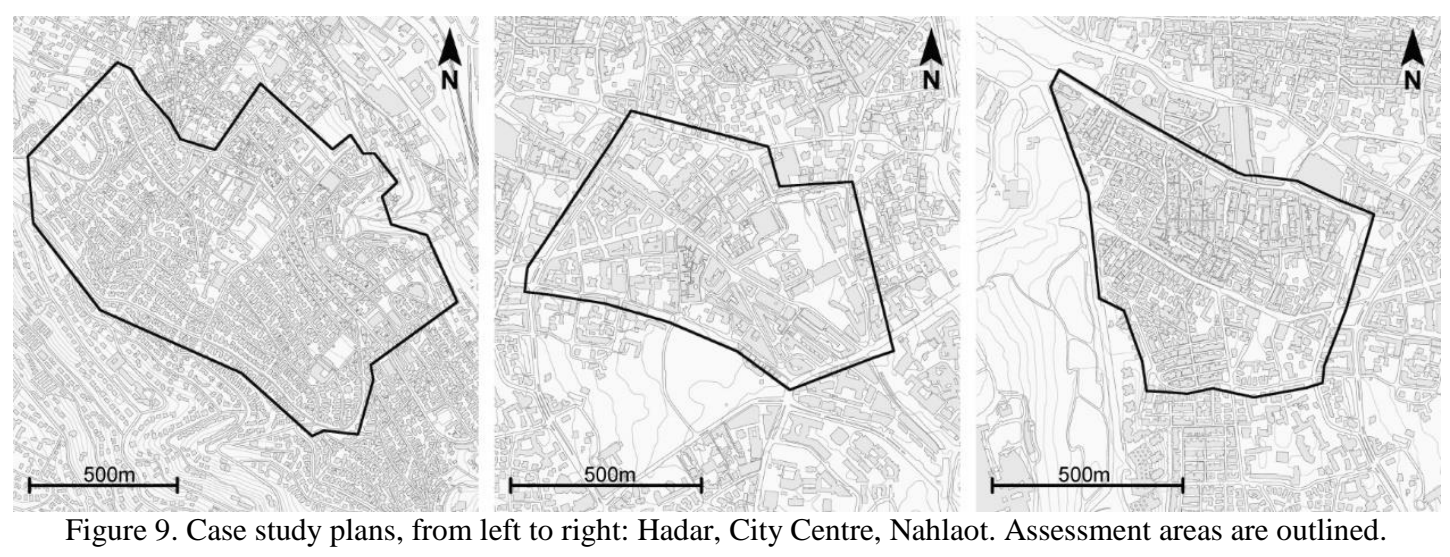

6 A summary of different physical and functional attributes of the case studies has been 7 compiled in table 1.

\begin{tabular}{|c|c|c|c|}
\hline \multirow{2}{*}{ Parameter } & Haifa & \multicolumn{2}{|c|}{ Jerusalem } \\
\cline { 2 - 4 } & 1 - Hadar & 2 - City Center & 3 - Nahlaot \\
\hline Regular segments & 404 & 266 & 396 \\
\hline Stair segments & 53 & 11 & 35 \\
\hline Regular buffer segments & 695 & 1714 & 1617 \\
\hline Stair buffer segments & 61 & 82 & 73 \\
\hline Total segments & 1213 & 2073 & 2121 \\
\hline Avg. segment length & $52.75 \mathrm{~m}$ & $43.69 \mathrm{~m}$ & $35.62 \mathrm{~m}$ \\
\hline Average slope & $6.12 \%$ & $3.69 \%$ & $4.07 \%$ \\
\hline No. of businesses & 2168 & 1295 & 497 \\
\hline Business/segment & 4.74 & 4.68 & 1.15 \\
\hline Avg. business density & 0.09 & 0.11 & 0.03 \\
\hline
\end{tabular}

9 All case studies were chosen based on their pre-modern, non-hierarchical, street-based 10 planning to avoid the inconsistencies in relations between centrality measures, 11 pedestrian movement volumes and retail locations that often occur in modernist 12 planned neighbourhoods (Omer, Rofè and Lerman, 2015). 

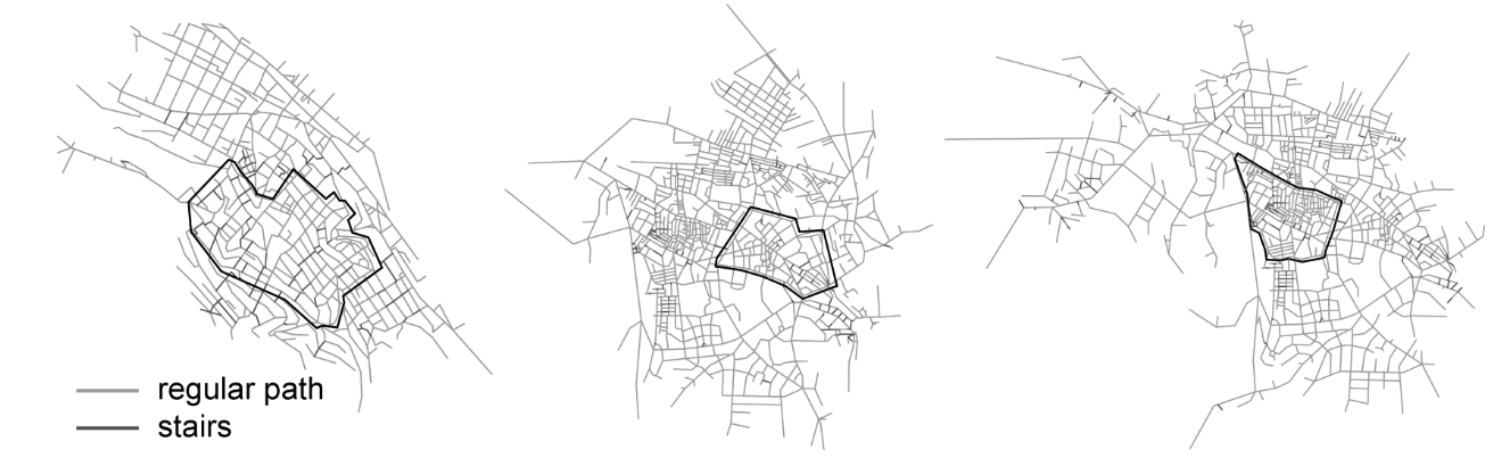

Figure 10. Case study segment maps, including the 'buffer' (outside the black line): Hadar, City Centre and Nahlaot.

\section{$4 \quad$ 5. Results}

The case studies were analysed for integration and the results were correlated against business density. Table 2 sums up the results of this analysis and contains average integration scores for the cases, in search of a global pattern. Maps of global

8 integration analysis for all cases appear in Figure 11.

9 All models performed reasonably well in global integration analysis, except for the 10 angular model in case 3.

\begin{tabular}{|c|c|c|c|c|c|c|c|}
\hline \multicolumn{2}{|c|}{ Model } & \multicolumn{2}{c|}{ Topological } & \multicolumn{2}{c|}{ Angular } & \multicolumn{2}{c|}{ PEM } \\
\cline { 3 - 8 } & & r & Avg. int. & r & Avg. int. & r & Avg. int. \\
\hline \multirow{3}{*}{ 1 - Hadar } & global & 0.486 & 0.076 & 0.488 & 0.116 & 0.49 & 0.074 \\
\cline { 2 - 8 } & $\mathbf{r}=\mathbf{3}$ & -0.27 & 0.446 & -0.143 & 0.536 & -0.201 & 0.579 \\
\cline { 2 - 8 } & $\mathbf{r}=4$ & -0.174 & 0.337 & 0.071 & 0.381 & -0.268 & 0.406 \\
\hline \multirow{3}{*}{ 2- City Centre } & global & 0.469 & 0.067 & 0.444 & 0.1 & 0.468 & 0.066 \\
\cline { 2 - 8 } & $\mathbf{r}=\mathbf{3}$ & -0.05 & 0.44 & -0.095 & 0.53 & -0.157 & 0.565 \\
\cline { 2 - 8 } & $\mathbf{r}=\mathbf{4}$ & 0.082 & 0.334 & 0.152 & 0.38 & -0.052 & 0.398 \\
\hline \multirow{3}{*}{ 3 - Nahlaot } & global & 0.425 & 0.068 & 0.153 & 0.095 & 0.424 & 0.067 \\
\cline { 2 - 8 } & $\mathbf{r}=\mathbf{3}$ & -0.032 & 0.438 & -0.041 & 0.549 & -0.043 & 0.555 \\
\cline { 2 - 8 } & $\mathbf{r}=\mathbf{4}$ & 0.022 & 0.336 & 0.048 & 0.384 & 0.009 & 0.395 \\
\hline
\end{tabular}

Table 2. Correlation of integration values and business density and average integration values.

12 The radius analyses were less productive. Radius 3 and 4 were either meaningless or had weak, often negative, correlations. Radius 3 and 4 had weak negative correlations in case 1 (except radius 4 with the angular model) and no correlation in case 3 with all models. In case 2, radius 4 in the angular model had a weak positive correlation and radius 3 with the PEM had a weak negative correlation. In light of these insignificant results, only global integration analysis is discussed in chapter 6 . 
The PEM performed best in case 1, followed by the angular model. In case 2, the

2 topological model had the highest correlation, followed very closely by the PEM. Case

33 saw results roughly similar to case 2, albeit with slightly lesser correlation.

4 The average global integration for the PEM was always lower than that of the other 5 models, whereas the average integration in radius 3 and 4 analysis was higher. The

6 topological model, however, had the lowest average integration in analyses with radii.

7 The angular model had expectedly the highest average global integration, because it

8 tends to have lower weights for turns in systems with many oblique segments.

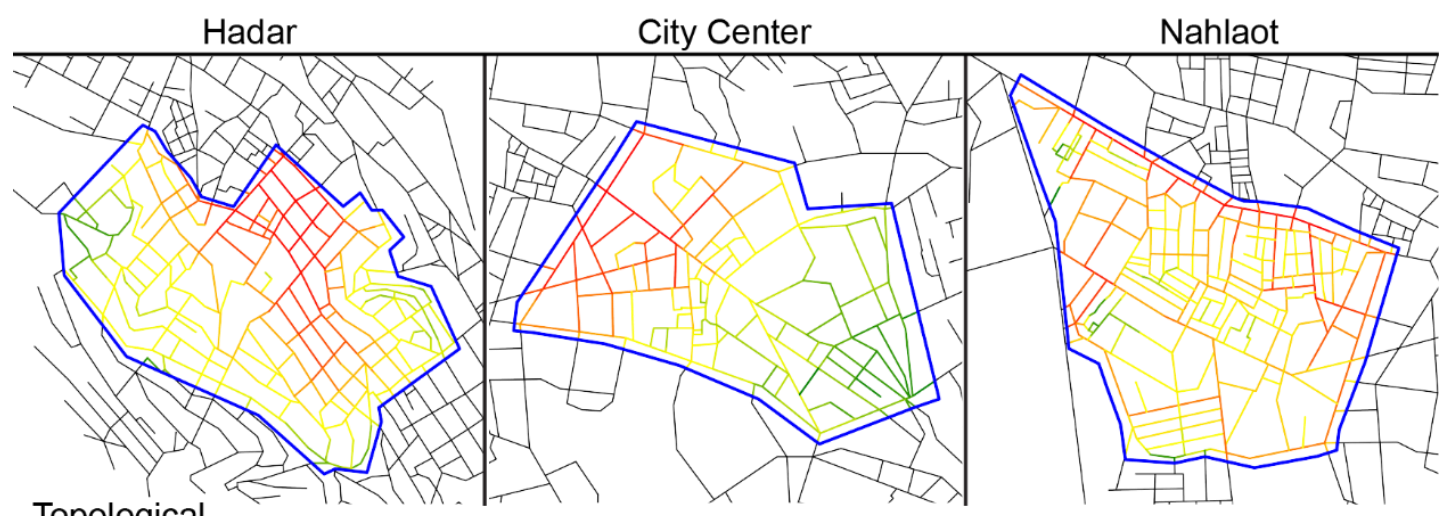

Topological

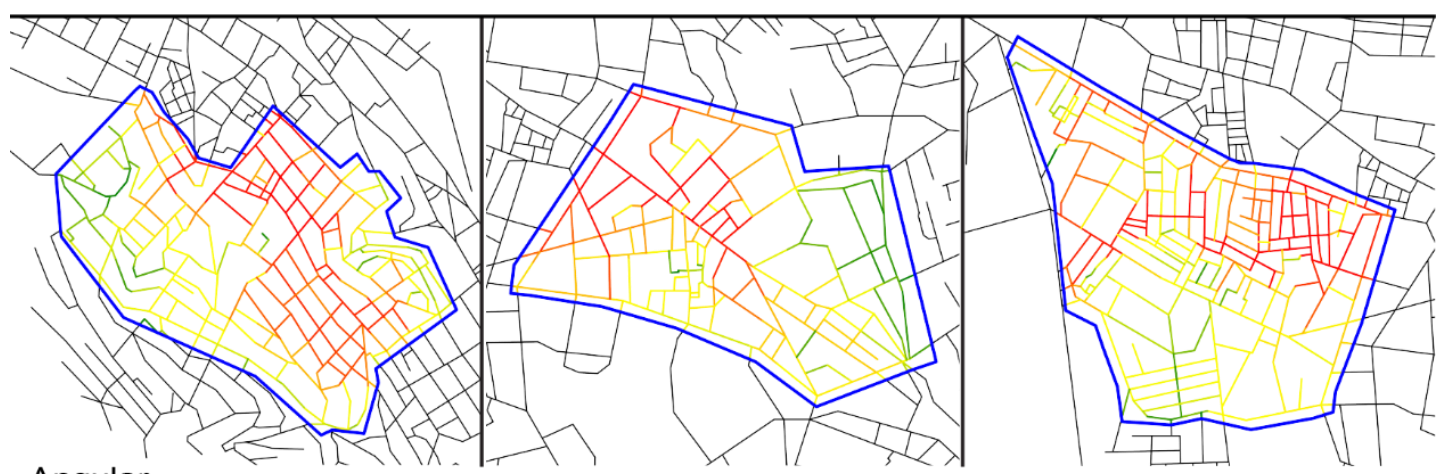

Angular

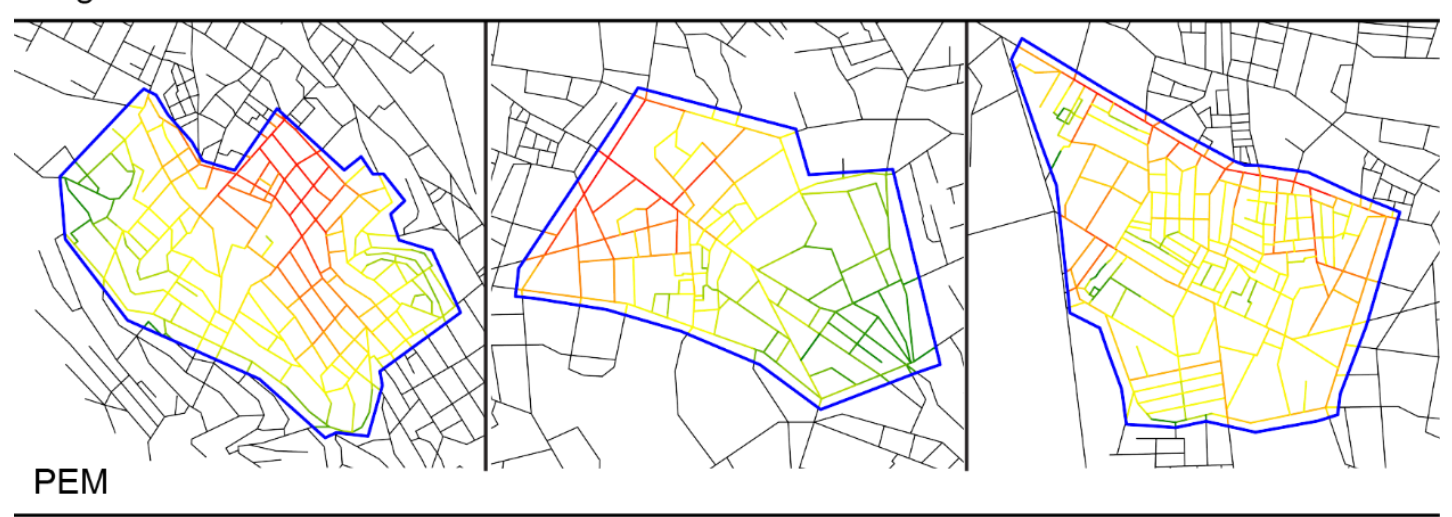




\section{Discussion}

The good performance of the PEM in case 1, Hadar, can be explained by the fact that it is the steepest of all cases and therefore potentially presents the PEM with a better platform to distinguish itself as its weighting system is based on slope.

All models performed generally better in cases 1 and 2, where the number of businesses and especially their density was the highest. Although all cases were sufficiently commercial to produce good results, this points at a possible weakness in the methodology and its limited applicability in less commercial areas.

Cases 2 and 3, City Centre and Nahlaot respectively, saw almost equal performance from the PEM and the Topological model, with the topological model having a slight edge. The relative similarity in performance suggests at least one of several options:

1) Basing of the PEM's weighting system on the Topological model has been over emphasized - giving insufficient emphasis to the effort-based weight.

2) The slope in some of the selected studies has not been significant enough to create a great divergence in results. This point is supported by a study by Sun et al (2015) where participants reported slopes of $5 \%$ and greater to be perceived as barriers to walking. The average slopes in cases 2 and 3 fall below that number.

Possibility 2 might be reinforced further by the fact that the topological model performed slightly better than the PEM in cases 2 and 3, since the addition of weights to insignificant slopes can degrade the performance of the model.

A possible explanation for the surprisingly bad performance of the angular model in case 3 might lie in the special geometric traits of Nahlaot: large parts of the test area are very dense with many narrow and winded alleys. This may cause retail and public functions to concentrate more in the larger main streets because they are the only ones that can support the required pedestrian volumes and supply vehicles. The angles between those streets and the neighbouring patches of dense alleys become less important in this context.

The consistently lower average global integration for the PEM might represent the general relative difficulty of walking on sloped terrain. The higher average integration 
1 in radius 3 and 4 analysis, on the other hand, is just the expected result of the weights

2 causing a fewer number of segments to be added to the local systems compared to the other models.

4 As a concluding remark, it should be remembered that a fundamental feature of the

5 PEM, the combination of two cognitive bases in one framework, is also the source of

6 a unique challenge. While it was shown that both visibility and physical effort affect

7 pedestrian movement decisions, the relationship between these two factors still

8 remains unclear (Montello, 1997). Answering questions about the dynamics of

9 different consideration and their relative importance will provide the PEM with better accuracy and theoretical grounding. This problem is complex enough to justify a separate research and calls for further empirical studies.

\section{Conclusions}

In this article, a novel analytical cognitive mapping method for pedestrian movement in sloped urban environment was presented.

Topography causes two important effects on pedestrian movement. First, topography can potentially block lines of sight and limit the spatial awareness of pedestrians. Second, the added effort of walking on steep slopes which can serve as a consideration for pedestrians when deciding which path to choose (or whether to walk at all).

A new model was developed to turn a $2 \mathrm{D}$ axial map into a $3 \mathrm{D}$ segment map that represents the topographic aspect of the environment. The suggested model is a hybrid in the sense that it combines two cognitive bases in one model: visibility and physical effort. The PEM deals with the problem of visual constraints stemming from topographic form by finding the points along a segment that block lines of sight and breaking the segment into shorter 3D segments. The problem of integrating the extra physical effort required to traverse a slope is tackled by attaching weights for ascent and descent to each 3D segment. One possible way of calculating the weights is proposed, but others that take physical effort into account directly are potentially valid. These two refinements provide a terrain-sensitive version of the axial segment map.

The initial assessment of the PEM was done by comparing it to two traditional models of the axial segment map, the topological model and the angular model. These methods 
are, however, two-dimensional and do not incorporate topographic information in their calculations.

The PEM proved its mettle in steep environments thus vindicating its own basic logic and proving the need for such a tool. However, further work is required in several directions. The weighting function of the PEM requires more adjustments. The problem of integrating two cognitive bases in a single framework also remains unsolved. A future validation study will include counts of actual pedestrian volumes and the construction of a pedestrian movement model. The results also make a case for the development of a PEM variant that will combine the traits of the physical and angular models, hopefully retaining the strengths of both models while negating the weaknesses.

The cognitive logic behind the PEM can also be implemented in models other than an axial segment map. For example, it can be used as a guideline in agent behavior in a future agent-based model.

Physical effort as a cognitive factor in pedestrian behavior is not limited to path selection. Other effects, such as variation in attention (Hutchinson \& Tenenbaum, 2006), can serve as a basis for other types of cognitive models.

The PEM can improve the ability of planners and designers to make informed decisions regarding the design of movement systems, public space and land-use allocation. Such a model will improve our understanding of pedestrian movement in urban environments and facilitate better human-centered design.

\section{Acknowledgements}

Data courtesy of SPaDeLib at the Center of Urban and Regional Studies in the Faculty of Architecture and Town Planning, Technion IIT and the GIS department of the Municipality of Jerusalem.

This research was supported by the Israel Science Foundation [grant number 1485/15], The Azrieli Foundation and The Irwin and Joan Jacobs Fellowship scholarship.

\section{References}


Al-Widyan F., Al-Ani A., Kirchner N., Zeibots M. (2017), 'An Effort-Based Evaluation of Pedestrian Route Selection', In Scientific Research and Essays, Vol. 12(4), p.42-50.

Asami, J., Kubat, A.S., Kitagawa, K., and Iida, S. (2003), 'Introducing the Third Dimension on Space Syntax: an Application on the Historical Istanbul', In: Hanson, J. (ed.), Proceedings of the Fourth International Space Syntax Symposium, London: University College London, p.48.1-48.18.

Bafna, S. (2003), 'Space Syntax: A Brief Introduction to Its' Logic and Analytical Techniques', In Environment and Behavior, Vol. 35, p.17-29.

Batty, M. (2001), 'Agent-Based Pedestrian Modeling', In Environment and Planning B: Planning and Design, Vol. 28, p.321-326.

Benedikt, M. (1979), 'To Take Hold of Space: Isovists and Isovist Fields', In Environment and Planning B, Vol. 6, p.47-65.

Chin, G.K.W., Van Niel, K.P., Giles-Corti, B. and Knuiman, M. (2007), 'Accessibility and Connectivity in Physical Activity Studies: The Impact of Missing Pedestrian Data', In Preventive Medicine, Vol. 46, p .41-45.

Conroy Dalton, R. (2003), 'The Secret is to Follow Your Nose: Route Path Selection and Angularity', In Environment \& Behavior, Vol. 35(1), p.107-131.

Conroy Dalton, R. and Dalton, N. S. (2007), 'Applying Depth Decay Functions to Space Syntax Network Graphs', In The Proceedings of the Sixth International Space Syntax Symposium, Istanbul, p. 89.1-89.13.

Conroy Dalton, R., Hölscher, C., and Turner A. (2012), ' Understanding Space: The Nascent Synthesis of Cognition and the Syntax of Spatial Morphologies', In Environment and Planning B: Planning and Design, Vol. 39, p.7-11.

Dalton, N. (2001), 'Fractional Configurational Analysis and a Solution to the Manhattan Problem'. In The Proceedings of the Third International Space Syntax Symposium, Atlanta.

Dijkstra, E.W. (1959) 'A Note on Two Problems in Connexion with Graphs', In Numerische Mathematik, Vol. 1, p.269-271.

Dutta, S. (1988), 'Approximate Spatial Reasoning', In M. Ali (ed.), Proceedings of the 1st International Conference on Industrial and Engineering Applications of Artificial Intelligence and Expert Systems. CRC Press, p. 126-140.

Egorova, E. (2018), 'Spatial Discourse Production: Applying Denis's Framework to Non-Urban Context', In: Creem-Regehr, S., Schöning, J. and Klippel, A. (eds.), Proceedings of Spatial Cognition 2018, Tübingen: Universität Tübingen, September 5-8, p.134-148.

Evans, G.W., Marrero, D.G. and Butler, P.A. (1981), 'Environmental Learning and Cognitive Mapping', In Environment and Behaviour, Vol. 13(1), p.83-104. 
Fisher-Gewirtzman, D. (2012), '3D Models as a Platform for Urban Analysis and Studies on Human Perception of Space', In: Rouch, P. and Wafa Skalli, W. (eds.), Proceedings of Usage, Usability and Utility of 3D City Models (3U3D2012), Nantes, 29-31 October, p.1-16.

Fisher-Gewirtzman, D. (2017), 'Can 3D Visibility Calculations Along a Path Predict the Perceived Density of Participants Immersed in a Virtual Reality Environment?', In: Heitor, T., Serra, M., Pinelo Silva, J., Bacharel, M., and Cannas da Silva, L. (eds.), Proceedings of the Eleventh International Space Syntax Symposium, Lisbon: Instituto Superior Técnico, 3-7 July, Vol. 5, p.160.1-160.18.

Fisher-Gewirtzman, D. and Natapov, A. (2014), 'Different Approaches of Visibility Analyses Applied on Hilly Urban Environment', In Survey Review, Vol. 388(46), p.366-382.

Fisher-Gewirtzman, D., Shashkov, A. and Doytsher, Y. (2013), 'Voxel Based Volumetric Visibility Analysis of Urban Environments', In Survey Review, Vol. 45(333), p.451-61.

Fisher-Gewirtzman, D. and Wagner, I. (2003), 'Spatial Openness as a Practical Metric for Evaluating Built- Up Environments', In Environment and Planning B: Planning and Design, Vol. 30(1), p.37-49.

Frith, M. J. (2017), 'Using Qualitative Distance Metrics in Space Syntax and Configurational Analyses', In: Heitor, T., Serra, M., Pinelo Silva, J., Bacharel, M., and Cannas da Silva, L. (eds.), Proceedings of the Eleventh International Space Syntax Symposium, Lisbon: Instituto Superior Técnico, 3-7 July, Vol. 5, p.161.1-161.15.

Gil, J. (2017), 'Street Network Analysis “Edge Effects'?: Examining the Sensitivity of Centrality Measures to Boundary Conditions', In Environment and Planning B: Urban Analytics and City Science, Vol. 44(5), p.819-836.

Golledge, R.G. (1995), 'Defining the Criteria Used in Path Selection', Berkeley, California: The University of California Transportation Center.

Greenberg E., Natapov A. and Fisher-Gewirtzman D., (2017), 'The Effect of Topography on Pedestrian Movement: Developing an Analytical Model', In: Heitor, T., Serra, M., Pinelo Silva, J., Bacharel, M., and Cannas da Silva, L. (eds.), Proceedings of the Eleventh International Space Syntax Symposium, Lisbon: Instituto Superior Técnico, 3-7 July, Vol. 6, p.198.1-198.6.

Hillier, B. (1996a), 'Cities and Movement Economies', In Urban Design International, Vol. 1(1), p.41-60.

Hillier, B. (1996b), Space is the Machine: A Configurational Theory of Architecture, Cambridge: Cambridge University Press.

Hillier, B. (1999), 'Centrality as a Process: Accounting for Attraction Inequalities in Deformed Grids', In Urban Design International, Vol. 4(3\&4), p.107-127. 
Hillier, B. and Hanson, J. (1984), The Social Logic of Space, Cambridge: Cambridge University Press.

Hillier, B., Penn, A., Hanson, J., Grajewski, T. and Xu, J. (1993), 'Natural Movement: Configuration and Attraction in Urban Pedestrian Movement', In Environment and Planning B: Planning and Design, Vol. 20, p.29-66.

Hutchinson, J. C. and Tenenbaum, G. (2007), 'Attention Focus during Physical Effort: The Mediating Role of Task Intensity', In Psychology of Sport and Exercise, Vol. 8, p. 233-245.

Jiang, B. and Claramunt, C. (2002), 'Integration of Space Syntax into GIS: New Perspectives for Urban Morphology', In Transactions in GIS, Vol. 6(3), p. 295-309.

Jiang, B. and Claramunt, C. (2004), 'Topological Analysis of Urban Street Networks', In Environment and Planning B, Vol. 31 (1). p. 151-162.

Jiang, B. (2005), 'A Structural Perspective on Visibility Patterns with a Topographic Surface', In Transactions in GIS, Vol. 9(4), p.475-488.

Jiang, B. (2006), 'Ranking Spaces for Predicting Human Movement in an Urban Environment', In International Journal of Geographical Information Science, Vol. 23(7), p.823-837.

Karimi, K. (2012), 'A Configurational Approach to Analytical Urban Design: "Space Syntax” Methodology', In Urban Design International, Vol. 17(4), p.297-318.

Kinsella-Shaw, J.M., Shaw, B. and Turvey, M.T. (1992) 'Perceiving "Walk-on-able" Slopes', In Ecological Psychology, Vol. 4(4), p.223-239.

Koutsolampros, P. and Varoudis, T. (2017), 'Assisted Agent-Based Simulations: Fusing Non-Player Character Movement with Space Syntax', In: Heitor, T., Serra, M., Pinelo Silva, J., Bacharel, M., and Cannas da Silva, L. (eds.), Proceedings of the Eleventh International Space Syntax Symposium, Lisbon: Instituto Superior Técnico, 3-7 July, Vol. 5, p.164.1-164.13.

Moar, I. and Bower G.H. (1983), 'Inconsistency in Spatial Knowledge', In Memory \& Cognition, Vol. 11(2), p.107-113.

Montello, D.R. (1998), 'A New Framework for Understanding the Acquisition of Spatial Knowledge in Large-Scale Environments', Egenhofer M.J. and Golledge R.G. (eds.), Spatial and Temporal Reasoning in Geographic Information Systems, p.143154.

Montello, D.R. (1991), 'Spatial Orientation and the Angularity of Urban Routes: A Field Study', In Environment and Behaviour, Vol. 23(1), p.47-69.

Montello, D.R. (1997), 'The Perception and Cognition of Environmental Distance: Direct Sources of Information', In Hirtle S.C. \& Frank A.U. (eds.), Spatial Information Theory: A Theoretical Basis for GIS, p.297-311. 
Montello, D.R. and Frank, A.U. (1996), 'Modeling Directional Knowledge and Reasoning in Environmental Space: Testing Qualitative Metrics', Mark D. \& White D. (eds.), The Construction of Cognitive Maps, p. 321-344.

Nourian, P., Van der Hoeven, F., Rezvani, S. and Sariyildiz, S. (2015), 'Easiest Paths for Walking and Cycling: Combining Syntactic and Geographic Analyses in Studying Walking and Cycling Mobility', In: Karimi, K., Vaughan, L., Sailer, K., Palaiologou, G. and Bolton, T. (eds.), Proceedings of the Tenth International Space Syntax Symposium, London: University College London, Vol. 4, p.78.1-78.15.

Okabe, A., Aoki, K. and Hamamoto, W. (1987), 'Distance and Direction Judgment in Large-Scale Natural Environments - Effects of a Slope and Winding Trail', In Environment and Behavior, Vol. 18(6), p.755-772.

Okabe, A. and Sugihara, K. (2012), 'Spatial Analysis along Networks: Statistical and Computational Methods', Chichester, West Sussex, John Wiley \& Sons.

Omer, I., Rofé, Y. and Lerman Y., (2015), 'The Impact of Planning on Pedestrian Movement: Contrasting Pedestrian Movement Models in Pre-modern and Modern Neighborhoods in Israel', In International Journal of Geographical Information Science, Vol. 29(12), p.2121-2142.

Penn, A., Hillier, B., Banister, D. and Xu, J., (1998), 'Configurational Modelling of Urban Movement Networks', In Environment and Planning B: Planning and Design, Vol. 25, p. 59-84.

Penn, A. and Turner, A. (2004), 'Movement-Generated Land-Use Agglomeration: Simulation Experiments on the Drivers of Fine-Scale Land-Use Patterning', In Urban Design International, Vol. 9, p. 81-96.

Pingel, T. J. (2010), 'Modeling Slope as a Contributor to Route Selection in Mountainous Areas', In Cartography and Geographic Information Science, Vol. 37(2), p. 137-148.

Proffitt, D.R., Stefanucci J., Banton T. \& Epstein W. (2003), 'The Role of Effort in Perceiving Distance', In Psychological Science, Vol. 14(2), p.106-112.

Ratti, C. (2004), 'Urban Texture and Space Syntax: Some Inconsistencies', In Environment and Planning B: Planning and Design, Vol. 31, p.487-499.

Sadalla, E.K. and Magel, S.G. (1980), 'The Perception of Traversed Distance', In Environment and Behaviour, Vol. 12(1), p.65-79.

Sadalla, E.K. and Montello, D.R. (1989), 'Remembering Changes in Direction', In Environment and Behaviour, Vol. 21(3), p.346-363.

Sadalla, E.K. and Staplin, L.J. (1980), 'The Perception of Traversed Distance: Intersections', In Environment and Behaviour, Vol. 12(2), p.167-182. 
Seneviratne, P. N. and Morral, J. F. (1985), 'Analysis of Factors Affecting the Choice of Route of Pedestrians', In Transportation Planning and Technology, Vol. 10, p. 147159 .

Sholl, M.J. (1987), 'Cognitive Maps as Orientation Schemata', In Journal of Experimental Psychology: Learning, Memory and Cognition, Vol. 13(4), p.615-628.

Stefanucci, J.K., Proffitt, D.R., Banton, T. and Epstein, W. (2005), 'Distances Appear Different on Hills', In Perception \& Psychophysics, Vol. 67(6), p.1052-1060.

Sun, G., Haining, R., Lin, H., Oreskovic, N.M. and He, J. (2015), 'Comparing the Perception with the Reality of Walking in a Hilly Environment: An Accessibility Method Applied to a University Campus in Hong Kong', In Geospatial Health, Vol. 10(1), p.32-39.

Thorndyke, P.W. (1981), 'Distance Estimation from Cognitive Maps', In Cognitive Psychology, Vol. 13, p.526-550.

Tobler, W. (1993), 'Three Presentations on Geographical Analysis and Modeling: Non-Isotropic Geographical Modeling Speculations on the Geometry of Geography Global Spatial Analysis', In National Center for Geographic Information and Analysis.

Turner, A., Doxa, M., O'Sullivan, D. and Penn, A. (2001), 'From Isovists to Visibility Graphs: A Methodology for the Analysis of Architectural Space', In Environment and Planning B: Planning and Design, Vol. 28(1), p.103-121.

Turner, A. (2001), 'Angular Analysis', In The Proceedings of the Third International Space Syntax Symposium, Atlanta, p. 30-31.

Turner, A. and Penn, A. (2002), 'Encoding Natural Movement as an Agent-Based System: An Investigation into Human Pedestrian Behavior in the Built Environment', In Environment and Planning B: Planning and Design, Vol. 29, p. 473-490.

Whitley, T. G. and Hicks, L. M. (2003), 'Geographic Information Systems Approach to Understanding Potential Prehistoric and Historic Travel Corridors', In Southeastern Archaeology, Vol. 22(1), p. 76-90.

Witt, J.K., Proffitt, D.R. and Epstein, W. (2010), 'When and How Are Spatial Perceptions Scaled?', In Journal of Experimental Psychology: Human Perception and Performance, Vol. 36(5), p.1153-1160.

Zadra, J.R., Weltman, A.L. and Proffitt, D.R. (2016), 'Walkable Distances Are Bioenergetically Scaled', In Journal of Experimental Psychology: Human Perception and Performance, Vol. 42(1), p.39-51.

Zadra, J.R. and Proffitt, D.R. (2016), 'Optic Flow is Calibrated to Walking Effort', In Psychonomic Bulletin \& Review, Vol. 23(5), p.1491-1496. 